\title{
Boundary value problem for nonlinear fractional differential equations with delay
}

\author{
Azmat Ullah Khan Niazi ${ }^{1 *}$, Jiang Wei ${ }^{1}$, Mujeeb Ur Rehman² and Pang Denghao
}

\author{
"Correspondence: \\ azmatmath@yahoo.com \\ 'School of Mathematical Sciences, \\ Anhui University, Hefei, Anhui \\ 230039, China \\ Full list of author information is \\ available at the end of the article
}

\begin{abstract}
We discuss the existence and uniqueness of a solution of a boundary value problem for a class of nonlinear fractional functional differential equations with delay involving Caputo fractional derivative. Our work relies on the Schauder fixed point theorem and contraction mapping principle in a cone. We also include examples to show the applicability of our results.
\end{abstract}

MSC: $34 \mathrm{~K} 37 ; 34 \mathrm{~B} 15$

Keywords: fractional functional differential equation; boundary value problem; existence and uniqueness of solutions

\section{Introduction}

Fractional calculus is an emerging field in applied mathematics that deals with derivatives and integrals of arbitrary orders and their applications. The original research started on this topic at the end of the seventeenth century. Fractional calculus helps us describing natural phenomena and mathematical models more accurately. Therefore, fractional differential equations are becoming more famous, and the theory and its applications have been greatly improved. For details, we refer to [1-5].

The existence and uniqueness of solutions to boundary value problems for fractional differential equations have gained these days interest of many authors. For details, we also refer to $[6,7]$, where the authors discuss existence results for coupled systems and uniqueness for $n$ th-order differential equations, respectively. Recently, much research has been done on boundary value problems of fractional ordinary differential equations [8-13] and initial value problems of fractional functional differential equations [14-19].

Naturally, the phenomenon of time-delay is so common and certain. Many changes and processes not only depend on the present status but also on the past status. However, the research on fractional functional differential equations with delay is relatively sparse [20-26]. Therefore, it is necessary to consider the time-delay effect in the mathematical modeling of fractional differential equations. However, there are relatively exiguous results dealing with boundary value problems of fractional functional differential equations with time delays. Mostly, researchers consider the case of implicit functions, so by keeping this in mind we consider neutral functions where the nonlinear function $f$ not only depends on $x_{t}$ but also depends on the fractional derivative $D_{0^{+}}^{\delta} x_{t}$ of the delayed term of the unknown function. Wherefore, in this paper, our aim is to study the existence and uniqueness of a

(c) The Author(s) 2017. This article is distributed under the terms of the Creative Commons Attribution 4.0 International License (http://creativecommons.org/licenses/by/4.0/), which permits unrestricted use, distribution, and reproduction in any medium, provided you give appropriate credit to the original author(s) and the source, provide a link to the Creative Commons license, and indicate if changes were made. 
solution of the following boundary value problem for a fractional differential equation:

$$
\left\{\begin{array}{l}
{ }^{\mathrm{c}} D_{0^{+}}^{\gamma} x(t)=f\left(t, x_{t},{ }^{\mathrm{c}} D_{0^{+}}^{\delta} x_{t}\right), \quad 0<t<1, \\
a x(t)+b x^{\prime}(t)=\zeta(t), \quad-\tau \leq t \leq 0 \\
c x(t)-d x^{\prime}(t)=\eta(t), \quad 1 \leq t \leq 1+\alpha
\end{array}\right.
$$

with $1<\gamma<2$ and $0<\delta<1, \zeta(0)=\eta(1)=0$, and $a, b, c, d \in \mathbb{R}$, where $a, b$ and $c, d$ are not simultaneously zero and such that $b c+a d-a c \neq 0$. For $0<\tau<1$, we denote by $C_{\tau}$ the Banach space of all continuous functions $\phi:[-\tau, \alpha] \rightarrow \mathbb{R}$ endowed with the supremum norm $\|\phi\|=\sup \{|\phi(s)| ;-\tau \leq s \leq \alpha\}$. If $X:[-\tau, 1] \rightarrow \mathbb{R}$, then, for any $t \in[0,1]$, we define $x_{t}$ by $x_{t}(\theta)=x(t+\theta)$ for $\theta \in[-\tau, 0]$, where $\tau, \alpha \geq 0$ are constants satisfying $0 \leq \tau+\alpha<1$, and

$$
C_{\tau}^{+}(0)=\left\{\phi \in C_{\tau} \mid \phi(\theta) \geq 0, \theta \in[-\tau, 0], \phi(0)=0,{ }^{\mathrm{c}} D^{\delta} \phi(s)=0\right\} .
$$

In addition, our work is inspired by three systems [11, 22, 27]. Li et al. [22] studied the existence of a solution of the following fractional differential equation involving the Caputo fractional derivative:

$$
\left\{\begin{array}{l}
{ }^{\mathrm{c}} D_{0^{+}}^{\alpha} u(t)=f\left(t, u_{t},{ }^{\mathrm{c}} D_{0^{+}}^{\beta} u(t)\right), \quad 0<t<1, \\
u^{\prime}(0)=0, \quad u^{\prime}(1)=\lambda u^{\prime}(\eta),
\end{array}\right.
$$

where ${ }^{\mathrm{c}} D_{0^{+}}^{\alpha}$ and ${ }^{\mathrm{c}} D_{0^{+}}^{\beta}$ are the Caputo fractional derivatives with $2<\alpha<3,0<\beta<1, \eta \in$ $(0,1)$, and $1<\lambda<\frac{1}{2 \eta}$.

Rehman et al. [11] studied the existence and uniqueness of a solution to a nonlinear three-point boundary value problem for the following fractional differential equation:

$$
\left\{\begin{array}{l}
{ }^{\mathrm{c}} D_{0^{+}}^{\delta} u(t)=f\left(t, u(t),{ }^{\mathrm{c}} D_{0^{+}}^{\sigma} u(t)\right), \quad 0 \leq t \leq 1 \\
u(0)=\alpha u(\eta), \quad u(1)=\beta u(\eta)
\end{array}\right.
$$

where $1<\delta<2,0<\sigma<1, \alpha, \beta \in \mathbb{R}, \alpha \eta(1-\beta)+(1-\alpha)(1-\beta \eta) \neq 0$, and $D_{0^{+}}^{\delta}$ and $D_{0^{+}}^{\sigma}$ denote the Caputo fractional derivatives. By the Banach contraction principle and Schauder fixed point theorem they obtained some new results on the existence and uniqueness.

Zhao and Wang [27] studied the existence and uniqueness of a solution to the integral boundary value problem for the nonlinear fractional differential equation

$$
\left\{\begin{array}{l}
{ }^{\mathrm{c}} D_{0^{+}}^{\alpha} u(t)+f\left(t, u_{t}, u^{\prime}(t),{ }^{\mathrm{c}} D_{0^{+}}^{\beta} u(t)\right)=0, \quad t \in J=(0,1] \\
u(0)-\gamma_{1} u(\lambda)=\delta_{1} \int_{0}^{\eta} u(s) d s, \\
u(1)-\gamma_{2} u(\lambda)=\delta_{2} \int_{0}^{\eta} u(s) d s, \quad u^{\prime \prime}(0)=0
\end{array}\right.
$$

where $0<\eta<1, \gamma_{1}, \gamma_{2}, \lambda_{1}, \lambda_{2}$ are nonnegative constants, and ${ }^{\mathrm{c}} D_{0^{+}}^{\alpha}$ and ${ }^{\mathrm{c}} D_{0^{+}}^{\beta}$ are the Caputo fractional derivatives of orders $2<\alpha<3$ and $0<\beta<1$. By the Banach contraction principle and Leray-Schauder-type theorems they have found out the existence of such type of functions.

To the best of our knowledge, it looks like nobody considered BVP (1). Therefore, we will find out the existence and uniqueness of a solution of the nonlinear BVP (1) under some further conditions. We consider the effect of time delays of both terms $x_{t}$ and $D_{0^{+}}^{\delta} x_{t}$, 
which was not considered in the literature [11, 22, 27]. Therefore, our study improves and extends the previous results in the relevant literature [11, 22, 27].

The paper is arranged as follows. In Section 2, we review some basic definitions and lemmas. Section 3 is devoted to the Green function and to the existence and uniqueness of the defined problem. In Section 4, we explain the existence and uniqueness of solutions using some examples.

\section{Preliminaries}

This part includes some basic definitions and results.

Definition 2.1 The gamma function is defined as

$$
\Gamma(\gamma)=\int_{0}^{\infty} e^{-t} t^{\gamma-1} d t, \quad \gamma>0
$$

One of the basic properties of the gamma function is that it satisfies the functional equation $\Gamma(\gamma+1)=\gamma \Gamma(\gamma)$.

Definition 2.2 The fractional integral for a function $f$ with lower limit $t_{0}$ and order $\gamma$ can be defined as

$$
I_{t_{0}^{+}}^{\gamma} f(t)=\frac{1}{\Gamma(\gamma)} \int_{t_{0}^{+}}^{t} \frac{f(s)}{(t-s)^{1-\gamma}} d s, \quad \gamma>0, t>t_{0}
$$

where the right-hand side of the equality is defined pointwise on $\mathbb{R}^{+}$.

Definition 2.3 The Caputo fractional derivative for a function $f:\left(t_{0},+\infty\right) \rightarrow \mathbb{R}$ of order $\gamma(n-1<\gamma<n)$ is given by

$$
{ }^{\mathrm{c}} D_{t_{0}^{+}}^{\gamma} f(t)=I_{t_{0}^{+}}^{n-\gamma} \frac{d^{n}}{d t^{n}} f(t)=\frac{1}{\Gamma(n-\gamma)} \int_{t_{0}^{+}}^{t} \frac{f^{(n)}(s)}{(t-s)^{\gamma+1-n}} d s, \quad t>t_{0},
$$

where $n=[\gamma]+1([\gamma]$ stands for the bracket function of $\gamma)$.

Definition 2.4 ([28]) Let $X$ be a real Banach space. A subset $K$ of $X$ is called a cone if the following are true:

(i) $K$ is nonempty closed, and $\{\theta, e\} \subset X$ (where $\theta$ is null, and $e$ is the multiplicative identity of the Banach space $X)$;

(ii) $a K+b K \subset K$ for any nonnegative $a, b$;

(iii) $K^{2}=K K \subset K$; and

(iv) $(K) \cap(-K)=\{\theta\}$.

Lemma 2.1 ([29]) Let $\gamma>0$. Then

$$
I_{t_{0}^{+}}^{\gamma \mathrm{c}} D_{t_{0}^{+}}^{\gamma} f(t)=f(t)+c_{0}+c_{1} t+c_{2} t^{2}+\cdots+c_{n-1} t^{n-1}
$$

for some $c_{i} \in \mathbb{R}, i=0,1,2, \ldots, n-1$, where $n=[\gamma]+1$. 
Lemma 2.2 (Leray-Schauder fixed point theorem [30]) Let B be a nonempty, bounded, closed, and convex subset of $a$ Banach space $X$, and let $P: B \rightarrow B$ be a compact and continuous map. Then $P$ has a fixed point in $B$.

Lemma 2.3 (The contraction mapping principle [30]) Let $T$ be a contraction on a Banach space $X$. Then $T$ has a unique fixed point in $X$, that is, there is a unique solution $x \in X$ to the equation

$$
T(x)=x .
$$

\section{Main results}

In this part, we find the Green function for the given BVP (1) and then discuss the existence and uniqueness of a solution by using the Schauder fixed point theorem and the Banach contraction principle.

Consider the system

$$
{ }^{\mathrm{c}} D_{0^{+}}^{\gamma} x(t)=u(t), \quad 0<t<1,
$$

with boundary conditions

$$
\left\{\begin{array}{l}
a x(t)+b x^{\prime}(t)=\zeta(t), \\
c x(t)-d x^{\prime}(t)=\eta(t), \\
\zeta(0)=\eta(1)=0
\end{array}\right.
$$

where $1<\gamma<2$, and $u:[0,1] \rightarrow \mathbb{R}$ is a differentiable function. Then we have the following:

Lemma 3.1 A function $x$ is a solution of equation (2) with boundary conditions (3) if and only if it has the form $x(t)=\int_{0}^{1} G(t, s) u(s) d s$, where

$$
G(t, s)=\left\{\begin{array}{cc}
\frac{1}{\Gamma(\gamma)}(t-s)^{\gamma-1}+\left(\frac{a t-b}{b c+a d-a c}\right)\left[\frac{c}{\Gamma(\gamma)} \int_{0}^{1}(1-s)^{\gamma-1} u(s) d s\right. & \\
\left.-\frac{d}{\Gamma(\gamma-1)} \int_{0}^{1}(1-s)^{\gamma-2} u(s) d s\right], & 0 \leq s \leq t \leq 1, \\
\left(\frac{a t-b}{b c+a d-a c}\right)\left[\frac{c}{\Gamma(\gamma)} \int_{0}^{1}(1-s)^{\gamma-1} u(s) d s\right. & \\
\left.-\frac{d}{\Gamma(\gamma-1)} \int_{0}^{1}(1-s)^{\gamma-2} u(s) d s\right], & 0 \leq t \leq s \leq 1 .
\end{array}\right.
$$

Proof Applying $I_{0^{+}}^{\gamma}$ to both sides of equation (2) and using Lemma (2.1), we have

$$
x(t)=I_{0^{+}}^{\gamma} u(t)+c_{1}+c_{2} t .
$$

Differentiating both sides w.r.t. $t$, we get

$$
x^{\prime}(t)=I_{0^{+}}^{\gamma-1} u(t)+c_{2}
$$

Using boundary conditions (3), we get $c_{1}=\frac{-b}{a} c_{2}$, so $c x(1)-d x^{\prime}(1)=0$ implies that

$$
\begin{aligned}
& c I_{0^{+}}^{\gamma} u(1)+c c_{1}+c c_{2}-d I_{0^{+}}^{\gamma-1} u(1)-d c_{2}=0, \\
& \frac{c}{\Gamma(\gamma)} \int_{0}^{1}(1-s)^{\gamma-1} u(s) d s-c \frac{b}{a} c_{2}+c c_{2}-\frac{d}{\Gamma(\gamma-1)} \int_{0}^{1}(1-s)^{\gamma-2} u(s) d s-d c_{2}=0,
\end{aligned}
$$




$$
c_{2}=\left(\frac{a}{b c+a d-a c}\right)\left[\frac{c}{\Gamma(\gamma)} \int_{0}^{1}(1-s)^{\gamma-1} u(s) d s-\frac{d}{\Gamma(\gamma-1)} \int_{0}^{1}(1-s)^{\gamma-2} u(s) d s\right] .
$$

Therefore,

$$
c_{1}=\left(\frac{-b}{b c+a d-a c}\right)\left[\frac{c}{\Gamma(\gamma)} \int_{0}^{1}(1-s)^{\gamma-1} u(s) d s-\frac{d}{\Gamma(\gamma-1)} \int_{0}^{1}(1-s)^{\gamma-2} u(s) d s\right] .
$$

Hence,

$$
\begin{aligned}
x(t)= & \frac{1}{\Gamma(\gamma)} \int_{0}^{t}(t-s)^{\gamma-1} u(s) d s+\left(\frac{a t-b}{b c+a d-a c}\right)\left[\frac{c}{\Gamma(\gamma)} \int_{0}^{1}(1-s)^{\gamma-1} u(s) d s\right. \\
& \left.-\frac{d}{\Gamma(\gamma-1)} \int_{0}^{1}(1-s)^{\gamma-2} u(s) d s\right] \\
= & \frac{1}{\Gamma(\gamma)} \int_{0}^{t}(t-s)^{\gamma-1} u(s) d s+\left(\frac{a t-b}{b c+a d-a c}\right)\left[\frac{c}{\Gamma(\gamma)} \int_{0}^{t}(1-s)^{\gamma-1} u(s) d s\right. \\
& \left.-\frac{d}{\Gamma(\gamma-1)} \int_{0}^{t}(1-s)^{\gamma-2} u(s) d s\right]+\left(\frac{a t-b}{b c+a d-a c}\right)\left[\frac{c}{\Gamma(\gamma)} \int_{t}^{1}(1-s)^{\gamma-1} u(s) d s\right. \\
& \left.-\frac{d}{\Gamma(\gamma-1)} \int_{t}^{1}(1-s)^{\gamma-2} u(s) d s\right] \\
= & \int_{0}^{1} G(t, s) u(s) d s .
\end{aligned}
$$

Here we can easily prove that the integral is also Caputo differentiable. Since

$$
\int_{0}^{1} G(t, s) u(s) d s=I_{0^{+}}^{\gamma-1} u(t)+c_{1}+c_{2} t
$$

where $c_{1}$ and $c_{2}$ are constants, $c_{1}+c_{2} t$ is Caputo differentiable, and $I_{0^{+}}^{\gamma-1} u(t)$ is also Caputo differentiable, the integral is Caputo differentiable.

For convenience, we define

$$
X=\left\{x \mid x \in C[-\tau, 1],{ }^{\mathrm{c}} D^{\delta} x \in C[-\tau, 1], 0<\delta<1\right\} .
$$

The space $X$ equipped with the norm

$$
\|x\|_{I}=\max _{t \in I}|x(t)|+\left.\max _{t \in I}\right|^{\mathrm{c}} D^{\delta} x(t) \mid, \quad \text { where } I=(0,1),
$$

is a Banach space. For $x_{0}=\phi$, by the definition of $x_{t}$ it follows that

$$
\begin{aligned}
& x_{t}=x(t+\theta), \\
& x_{0}=x(\theta)=\phi(\theta) \quad \text { for } \theta \in[-\tau, 0] .
\end{aligned}
$$

Thus, we have

$$
x(t)=\phi(t) \quad \text { for } t \in[-\tau, 0] .
$$


Since $f:[0,1] \times C_{\tau} \times C_{\tau} \rightarrow \mathbb{R}$ is a continuous function, from Lemma (3.1) we obtain that $x$ is a solution of BVP (1) if and only if it satisfies

$$
x(t)= \begin{cases}\int_{0}^{1} G(t, s) f\left(s, x_{s},{ }^{c} D^{\delta} x_{s}\right) d s, & t \in(0,1), \\ \phi(t), & t \in[-\tau, 0] .\end{cases}
$$

We define the operator $T: X \rightarrow X$ by

$$
T x(t)= \begin{cases}\int_{0}^{1} G(t, s) f\left(s, x_{s},{ }^{c} D^{\delta} x_{s}\right) d s, & t \in(0,1), \\ \phi(t), & t \in[-\tau, 0] .\end{cases}
$$

Define the constants

$$
\begin{aligned}
& M=\max _{0 \leq t \leq 1}\left(\int_{0}^{1}|G(t, s) m(s)| d s\right), \quad \text { where } m \in L[0,1], \\
& M^{*}=\max _{0 \leq t \leq 1}\left(\int_{0}^{1}\left|\frac{\partial}{\partial t} G(t, s) m(s)\right| d s\right), \quad \text { where } m \in L[0,1] \\
& N=\frac{a d-d(a-b) \gamma}{b c+a d-a c}+\frac{a c+c(b-a) \gamma}{(b c+a d-a c) \Gamma(2-\delta)}
\end{aligned}
$$

Theorem 3.1 Suppose that any one of the following is satisfied:

(C1) there exists a nonnegative function $m \in L[0,1]$ such that

$$
\left|f\left(t, x_{t},{ }^{\mathrm{c}} D_{0^{+}}^{\delta} x_{t}\right)\right| \leq m(t)+j\left\|x_{t}\right\|^{\psi_{1}}+k\left\|{ }^{\mathrm{c}} D_{0^{+}}^{\delta} x_{t}\right\|^{\psi_{2}}
$$

for $j, k \in \mathbb{R}^{+}$and $0<\psi_{1}, \psi_{2}<1$, or

(C2) there exists a nonnegative function $m \in L[0,1]$ such that

$$
\left|f\left(t, x_{t},{ }^{\mathrm{c}} D_{0^{+}}^{\delta} x_{t}\right)\right| \leq m(t)+j\left\|x_{t}\right\|^{\psi_{1}}+k\left\|{ }^{\mathrm{c}} D_{0^{+}}^{\delta} x_{t}\right\|^{\psi_{2}}
$$

for $j, k \in \mathbb{R}^{+}$and $\psi_{1}, \psi_{2}>1$.

Then BVP (1) has a solution.

Proof Assume that $(\mathrm{C} 1)$ holds and take

$$
v \geq \max \left\{3\left(M+\frac{M^{*}}{\Gamma(2-\delta)}\right),(3 j N)^{\frac{1}{1-\psi_{1}}},(3 k N)^{\frac{1}{1-\psi_{2}}}\right\} .
$$

Let $K:=\left\{x \in X:\left\|x_{s}\right\| \leq v,\left\|^{c} D^{\delta} x_{s}\right\| \leq v, v>0, s \in(-\tau, \alpha)\right\}$. Then by definition (2.4) we can easily prove that $K$ is a cone. For any $x \in X$, we obtain

$$
\begin{aligned}
|T x(t)| & =\left|\int_{0}^{1} G(t, s) f\left(s, x_{s},{ }^{\mathrm{c}} D_{0^{+}}^{\delta} x_{s}\right) d s\right| \\
& \leq \int_{0}^{1}|G(t, s)| f\left(s, x_{s},{ }^{\mathrm{c}} D_{0^{+}}^{\delta} x_{s}\right) \mid d s \\
& \leq \int_{0}^{1}|G(t, s)|\left(m(s)+j\left\|x_{s}\right\|^{\psi_{1}}+k\|\|^{\mathrm{c}} D_{0^{+}}^{\delta} x_{s} \|^{\psi_{2}}\right) d s \\
& \leq \int_{0}^{1}|G(t, s) m(s)| d s+\int_{0}^{1}\left(j\left\|x_{s}\right\|^{\psi_{1}}+k\left\|{ }^{\mathrm{c}} D_{0^{+}}^{\delta} x_{s}\right\|^{\psi_{2}}\right) G(t, s) d s .
\end{aligned}
$$


Since $f$ is continuous on $(0,1)$, by the mean value theorem there exists $v$ such that

$$
\begin{aligned}
|T x(t)| \leq & M+j\|v\|^{\psi_{1}}+k\|v\|^{\psi_{2}}\left[\frac{1}{\Gamma(\gamma)} \int_{0}^{t}(t-s)^{\gamma-1} d s+\left(\frac{a t-b}{b c+a d-a c}\right)\right. \\
& \left.\times\left\{\frac{c}{\Gamma(\gamma)} \int_{0}^{1}(1-s)^{\gamma-1} d s-\frac{d}{\Gamma(\gamma-1)} \int_{0}^{1}(1-s)^{\gamma-2} d s\right\}\right] \\
\leq & M+\frac{j\|v\|^{\psi_{1}}+k\|v\|^{\psi_{2}}}{\Gamma(\gamma)}\left[\frac{t^{\gamma}}{\gamma}+\frac{a t-b}{b c+a d-a c}\left(\frac{c}{\gamma}-d\right)\right] .
\end{aligned}
$$

So,

$$
|T x(t)| \leq M+\frac{j\|v\|^{\psi_{1}}+k\|v\|^{\psi_{2}}}{\Gamma(\gamma+1)}\left[\frac{a d(1-\gamma)+\gamma b d}{b c+a d-a c}\right] .
$$

Also,

$$
\begin{aligned}
\left|T x^{\prime}(t)\right| \leq & \int_{0}^{1}\left|\frac{\partial}{\partial t} G(t, s)\right|\left|f\left(s, x_{s},{ }^{c} D_{0^{+}}^{\delta} x_{s}\right)\right| d s \\
\leq & \int_{0}^{1}\left|\frac{\partial}{\partial t} G(t, s)\right|\left(m(s)+j\left\|x_{s}\right\|^{\psi_{1}}+k\left\|{ }^{\mathrm{c}} D_{0^{+}}^{\delta} x_{s}\right\|^{\psi_{2}}\right) d s \\
\leq & \int_{0}^{1}\left|\frac{\partial}{\partial t} G(t, s) m(s)\right| d s+\left(j\|v\|^{\psi_{1}}+k\|v\|^{\psi_{2}}\right)\left[\frac{1}{\Gamma(\gamma-1)} \int_{0}^{t}(t-s)^{\gamma-2} d s\right. \\
& \left.+\left(\frac{a}{b c+a d-a c}\right)\left\{\frac{c}{\Gamma(\gamma)} \int_{0}^{1}(1-s)^{\gamma-1} d s-\frac{d}{\Gamma(\gamma-1)} \int_{0}^{1}(1-s)^{\gamma-2} d s\right\}\right] \\
\leq & M^{*}+\frac{j\|v\|^{\psi_{1}}+k\|v\|^{\psi_{2}}}{\Gamma(\gamma)}\left[t^{\gamma-1}+\frac{a}{b c+a d-a c}\left(\frac{c}{\gamma}-d\right)\right] .
\end{aligned}
$$

Therefore,

$$
\left|T x^{\prime}(t)\right| \leq M^{*}+\frac{j\|v\|^{\psi_{1}}+k\|v\|^{\psi_{2}}}{\Gamma(\gamma+1)}\left[\frac{b c \gamma+a c(1-\gamma)}{b c+a d-a c}\right] .
$$

Hence,

$$
\begin{aligned}
\left|{ }^{\mathrm{c}} D_{0^{+}}^{\delta} T x(t)\right| & \leq \frac{1}{\Gamma(1-\delta)} \int_{0}^{t}(t-s)^{-\delta}\left|T x^{\prime}(s)\right| d s \\
& \leq \frac{1}{\Gamma(1-\delta)} \frac{t^{1-\delta}}{1-\delta}\left[M^{*}+\frac{j\|v\|^{\psi_{1}}+k\|v\|^{\psi_{2}}}{\Gamma(\gamma+1)}\left(\frac{b c \gamma+a c(1-\gamma)}{b c+a d-a c}\right)\right] \\
& \leq \frac{M^{*}}{\Gamma(2-\delta)}+\frac{j\|v\|^{\psi_{1}}+k\|v\|^{\psi_{2}}}{\Gamma(\gamma+1) \Gamma(2-\delta)}\left(\frac{b c \gamma+a c(1-\gamma)}{b c+a d-a c}\right) .
\end{aligned}
$$

So by equation (5) we have

$$
\begin{aligned}
\|T x(t)\| \leq & M+\frac{j\|v\|^{\psi_{1}}+k\|v\|^{\psi_{2}}}{\Gamma(\gamma+1)}\left[\frac{a d(1-\gamma)+\gamma b d}{b c+a d-a c}\right]+\frac{M^{*}}{\Gamma(2-\delta)} \\
& +\frac{j\|v\|^{\psi_{1}}+k\|v\|^{\psi_{2}}}{\Gamma(\gamma+1) \Gamma(2-\delta)}\left(\frac{b c \gamma+a c(1-\gamma)}{b c+a d-a c}\right)
\end{aligned}
$$




$$
\begin{aligned}
\leq & M+\frac{M^{*}}{\Gamma(2-\delta)}+\frac{j\|v\|^{\psi_{1}}+k\|v\|^{\psi_{2}}}{\Gamma(\gamma+1)}\left[\frac{a d-d(a-b) \gamma}{b c+a d-a c}\right. \\
& \left.+\frac{a c+c(b-a) \gamma}{b c+a d-a c \Gamma(2-\delta)}\right] \\
\leq & \frac{v}{3}+\left(j\|v\|^{\psi_{1}}+k\|v\|^{\psi_{2}}\right) N \\
\leq & \frac{v}{3}+\frac{v}{3}+\frac{v}{3} \leq v .
\end{aligned}
$$

Thus, $T: K \rightarrow K$. Hence, $T$ is continuous since $f$ and $G$ are continuous. Now if (C2) holds, then we choose

$$
v \leq \min \left\{3\left(M+\frac{M^{*}}{\Gamma(2-\delta)}\right),(3 j N)^{\frac{1}{1-\psi_{1}}},(3 k N)^{\frac{1}{1-\psi_{2}}}\right\}
$$

and by the same process as before we obtain $\|T x(t)\| \leq \nu$, which implies that $T: K \rightarrow K$.

Now we will prove that $T$ is a completely continuous operator.

Let $P:=\max _{0 \leq t \leq 1}\left|f\left(t, x_{t},{ }^{\mathrm{c}} D_{0^{+}}^{\delta} x_{t}\right)\right|+1$. Then, for any $x \in X$ and $t_{1}, t_{2} \in[-\tau, 1]$ with $t_{1}<t_{2}$, by Lemma(3.1), for $0 \leq t_{1} \leq t_{2} \leq 1$, we have

$$
\begin{aligned}
\mid & T x\left(t_{2}\right)-T x\left(t_{1}\right) \mid \\
\leq & \int_{0}^{t_{1}}\left|G\left(t_{2}, s\right)-G\left(t_{1}, s\right)\right| P d s+\int_{t_{2}}^{1}\left|G\left(t_{2}, s\right)-G\left(t_{1}, s\right)\right| P d s \\
& +\int_{t_{1}}^{t_{2}}\left|G\left(t_{2}, s\right)-G\left(t_{1}, s\right)\right| P d s \\
\leq & P \mid \frac{1}{\Gamma(\gamma)}\left(\int_{0}^{t_{1}}\left(t_{1}-s\right)^{\gamma-1} d s-\int_{0}^{t_{2}}\left(t_{2}-s\right)^{\gamma-1} d s\right) \\
& +\frac{a\left(t_{2}-t_{1}\right)}{b c+a d-a c}\left(\frac{c}{\Gamma(\gamma)} \int_{0}^{1}(1-s)^{\gamma-1} d s-\frac{d}{\Gamma(\gamma-1)} \int_{0}^{1}(1-s)^{\gamma-2} d s\right) \mid \\
\leq & \frac{P}{\Gamma(\gamma+1)}\left[\left(t_{2}^{\gamma}-t_{1}^{\gamma}\right)+\frac{a\left(t_{2}-t_{1}\right)(c-d \gamma)}{b c+a d-a c}\right]
\end{aligned}
$$

and

$$
\begin{aligned}
&\left|{ }^{\mathrm{c}} D_{0^{+}}^{\delta} T x\left(t_{2}\right)-{ }^{\mathrm{c}} D_{0^{+}}^{\delta} T x\left(t_{1}\right)\right| \\
&=\frac{1}{\Gamma(1-\delta)}\left|\int_{0}^{t_{2}}\left(t_{2}-s\right)^{-\delta} T x^{\prime}(s) d s-\int_{0}^{t_{1}}\left(t_{1}-s\right)^{-\delta} T x^{\prime}(s) d s\right| \\
& \leq \frac{1}{\Gamma(1-\delta)}\left|\int_{0}^{t_{2}}\left(t_{2}-s\right)^{-\delta} T x^{\prime}(s) d s-\int_{0}^{t_{1}}\left(t_{2}-s\right)^{-\delta} T x^{\prime}(s) d s\right| \\
& \quad+\frac{1}{\Gamma(1-\delta)}\left|\int_{0}^{t_{1}}\left(t_{2}-s\right)^{-\delta} T x^{\prime}(s) d s-\int_{0}^{t_{1}}\left(t_{1}-s\right)^{-\delta} T x^{\prime}(s) d s\right| \\
& \leq \frac{1}{\Gamma(1-\delta)}\left(\int_{t_{1}}^{t_{2}}\left(t_{2}-s\right)^{-\delta}\left|T x^{\prime}(s)\right| d s+\int_{0}^{t_{1}}\left(\left(t_{2}-s\right)^{-\delta}-\left(t_{1}-s\right)^{-\delta}\right)\left|T x^{\prime}(s)\right| d s\right) \\
& \leq \frac{1}{\Gamma(1-\delta)}\left(\int_{t_{1}}^{t_{2}}\left(t_{2}-s\right)^{-\delta}\left(\int_{0}^{1}\left|\frac{\partial}{\partial t} G(t, s)\right| f\left(t, x_{t},{ }^{\mathrm{c}} D_{0^{+}}^{\delta} x_{t}\right) \mid d t\right) d s\right)
\end{aligned}
$$




$$
\begin{aligned}
& +\int_{0}^{t_{1}}\left[\left(t_{2}-s\right)^{-\delta}-\left(t_{1}-s\right)^{-\delta}\right]\left(\int_{0}^{1}\left|\frac{\partial}{\partial t} G(t, s)\right|\left|f\left(t, x_{t},{ }^{\mathrm{c}} D_{0^{+}}^{\delta} x_{t}\right)\right| d t\right) d s \\
\leq & \frac{P(b c \gamma+a c-a c \gamma)}{(b c+a d-a c) \Gamma(2-\delta) \Gamma(\gamma+1)}\left|t_{2}^{1-\delta}-t_{1}^{1-\delta}\right| .
\end{aligned}
$$

Hence, for $0 \leq t_{1}<t_{2} \leq 1$, we have

$$
\begin{aligned}
\left\|T x\left(t_{2}\right)-T x\left(t_{1}\right)\right\| \leq & \frac{P}{\Gamma(\gamma+1)}\left[\left|\left(t_{2}^{\gamma}-t_{1}^{\gamma}\right)+\frac{a\left(t_{2}-t_{1}\right)(c-d \gamma)}{b c+a d-a c}\right|\right. \\
& \left.+\left|\frac{(b c \gamma+a c-a c \gamma)}{(b c+a d-a c) \Gamma(2-\delta)}\left(t_{2}^{1-\delta}-t_{1}^{1-\delta}\right)\right|\right] .
\end{aligned}
$$

If $-\tau \leq t_{1}<0<t_{2} \leq 1$, then

$$
\begin{aligned}
\left|T x\left(t_{2}\right)-T x\left(t_{1}\right)\right| \leq & \left|T x\left(t_{2}\right)-T x(0)\right|+\left|T x(0)-T x\left(t_{1}\right)\right| \\
\leq & \int_{0}^{1}\left|G\left(t_{2}, s\right)-G(0, s)\right| d s+\left|\phi(0)-\phi\left(t_{1}\right)\right| \\
\leq & \mid \frac{P}{\Gamma(\gamma)} \int_{0}^{t_{2}}\left(t_{2}-s\right)^{\gamma-1} d s+\frac{a t_{2}}{b c+a d-a c} \\
& \times\left(\frac{c}{\Gamma(\gamma)} \int_{0}^{1}(1-s)^{\gamma-1} d s-\frac{d}{\Gamma(\gamma-1)} \int_{0}^{1}(1-s)^{\gamma-2} d s\right) \mid+\phi\left(t_{1}\right) \\
\leq & \frac{P}{\Gamma(\gamma)}\left|\frac{t_{2}^{\gamma}}{\gamma}+\frac{a t_{2}}{b c+a d-a c}\left(\frac{c}{\gamma}-d\right)\right|+\phi\left(t_{1}\right) .
\end{aligned}
$$

Also, if $-\tau \leq t_{1}<0<t_{2} \leq 1$, then

$$
\begin{aligned}
& \left|{ }^{\mathrm{c}} D_{0^{+}}^{\delta} T x\left(t_{2}\right)-{ }^{\mathrm{c}} D_{0^{+}}^{\delta} T x\left(t_{1}\right)\right| \\
& \left.\quad=\frac{1}{\Gamma(1-\delta)}\left|\int_{0}^{t_{2}}\left(t_{2}-s\right)^{-\delta} T x^{\prime}(s) d s\right|-0 \quad \text { (by the definition of } C_{\tau}^{+}(0)\right) \\
& \quad \leq \frac{P}{\Gamma(1-\delta)}\left(\frac{t_{2}^{1-\delta}}{1-\delta}\right) .
\end{aligned}
$$

Hence, if $-\tau \leq t_{1}<0<t_{2} \leq 1$, then

$$
\left\|T x\left(t_{2}\right)-T x\left(t_{1}\right)\right\| \leq\left|\frac{P}{\Gamma(\gamma)}\left(\frac{t_{2}^{\gamma}}{\gamma}+\frac{a t_{2}}{b c+a d-a c}\left(\frac{c}{\gamma}-d\right)\right)\right|+\left\|\phi\left(t_{1}\right)\right\|+\left|\frac{P t_{2}^{1-\delta}}{\Gamma(2-\delta)}\right| .
$$

If $-r \leq t_{1}<t_{2} \leq 0$, then from the definition of $\phi$ we get

$$
\left|T x\left(t_{2}\right)-T x\left(t_{1}\right)\right|=\left|\phi\left(t_{2}\right)-\phi\left(t_{1}\right)\right|
$$

and also

$$
\left|{ }^{\mathrm{c}} D_{0^{+}}^{\delta} T x\left(t_{2}\right)-{ }^{\mathrm{c}} D_{0^{+}}^{\delta} T x\left(t_{1}\right)\right|=\left|{ }^{\mathrm{c}} D_{0^{+}}^{\delta} \phi\left(t_{2}\right)-{ }^{\mathrm{c}} D_{0^{+}}^{\delta} \phi\left(t_{1}\right)\right|=0 .
$$

So

$$
\left\|T x\left(t_{2}\right)-T x\left(t_{1}\right)\right\|=\left\|\phi\left(t_{2}\right)-\phi\left(t_{1}\right)\right\| .
$$


Hence, by the preceding we can say that $\left\|T x\left(t_{2}\right)-T x\left(t_{1}\right)\right\| \rightarrow 0$ as $t_{2} \rightarrow t_{1}$, that is, for any $\epsilon>0$, there exists $\delta>0$ independent of $t_{1}, t_{2}$ and $x$ such that $\left|T x\left(t_{2}\right)-T x\left(t_{1}\right)\right| \leq \epsilon$ whenever $\left|t_{2}-t_{1}\right|<\delta$. Therefore, $T: X \rightarrow X$ is completely continuous.

Now we will use the Banach contraction principle to prove the uniqueness of the solution. For convenience, we denote

$$
\begin{aligned}
& L:=\frac{1}{\Gamma(\gamma+1)}\left[\frac{a d(1-\gamma)+\gamma b d}{b c+a d-a c}\right], \\
& L^{*}:=\frac{1}{\Gamma(2-\delta) \Gamma(\gamma+1)}\left[\frac{b c \gamma+a c(1-\gamma)}{b c+a d-a c}\right] .
\end{aligned}
$$

Theorem 3.2 Suppose that

$$
\left|f\left(t, x_{1}, x_{2}\right)-f\left(t, \bar{x}_{1}, \bar{x}_{2}\right)\right| \leq q\left(\left|x_{1}-\bar{x}_{1}\right|+\left|x_{2}-\bar{x}_{2}\right|\right)
$$

for each $0<t<1$ and all $x_{1}, x_{2}, \bar{x}_{1}, \bar{x}_{2} \in C_{\tau}$, where $q$ is a positive constant. If $q<\left(L+L^{*}\right)^{-1}$, then BVP (1) has a unique solution.

Proof From the definition of $T$ we get

$$
\begin{aligned}
|T x(t)-T \bar{x}(t)| \leq & \int_{0}^{1}|G(t, s)|\left|f\left(s, x_{s},{ }^{c} D_{0^{+}}^{\delta} x_{s}\right)-f\left(s, \bar{x}_{s},{ }^{c} D_{0^{+}}^{\delta} \bar{x}_{s}\right) d s\right| \\
\leq & q\|x-\bar{x}\|\left[\frac{1}{\Gamma(\gamma)} \int_{0}^{t}(t-s)^{\gamma-1} d s+\left(\frac{a t-b}{b c+a d-a c}\right)\right. \\
& \left.\times\left\{\frac{c}{\Gamma(\gamma)} \int_{0}^{1}(1-s)^{\gamma-1} d s-\frac{d}{\Gamma(\gamma-1)} \int_{0}^{1}(1-s)^{\gamma-2} d s\right\}\right] \\
\leq & \frac{q\|x-\bar{x}\|}{\Gamma(\gamma)}\left[\frac{t^{\gamma}}{\gamma}+\frac{a t-b}{b c+a d-a c}\left(\frac{c}{\gamma}-d\right)\right] \\
\leq & \frac{q\|x-\bar{x}\|}{\Gamma(\gamma+1)}\left[\frac{a d(1-\gamma)+\gamma b d}{b c+a d-a c}\right] \\
\leq & q\|x-\bar{x}\| L .
\end{aligned}
$$

Also,

$$
\begin{aligned}
\left|{ }^{\mathrm{c}} D_{0^{+}}^{\delta} T x(t)-{ }^{\mathrm{c}} D_{0^{+}}^{\delta} T \bar{x}(t)\right| \leq & \left|\frac{1}{\Gamma(1-\delta)} \int_{0}^{t}(t-s)^{-\delta}\left((T x)^{\prime}(s)-(T \bar{x})^{\prime}(s) d s\right)\right| \\
\leq & \frac{1}{\Gamma(1-\delta)} \int_{0}^{t}(t-s)^{-\delta}\left(\int_{0}^{1}\left|\frac{\partial}{\partial s} G(s, p)\right| \mid f\left(p, x_{p},{ }^{\mathrm{c}} D_{0^{+}}^{\delta} x_{p}\right)\right. \\
& \left.-f\left(p, \bar{x}_{p},{ }^{\mathrm{c}} D_{0^{+}}^{\delta} \bar{x}_{p}\right) \mid d p\right) d s \\
\leq & \frac{q\|x-\bar{x}\|}{\Gamma(1-\delta)} \int_{0}^{t}(t-s)^{-\delta}\left(\int_{0}^{t}\left|\frac{\partial}{\partial s} G(s, p)\right| d p\right) d s .
\end{aligned}
$$


Now by the definition of $G(t, s)$ we have

$$
\begin{aligned}
\int_{0}^{1}\left|\frac{\partial}{\partial t} G(t, s)\right| d s \leq & \frac{1}{\Gamma(\gamma-1)} \int_{0}^{t}\left|(t-s)^{\gamma-2}\right| d s+\left(\frac{a}{b c+a d-a c}\right) \\
& \times\left\{\frac{c}{\Gamma(\gamma)} \int_{0}^{1}\left|(1-s)^{\gamma-1}\right| d s-\frac{d}{\Gamma(\gamma-1)} \int_{0}^{1}\left|(1-s)^{\gamma-2}\right| d s\right\} \\
\leq & \frac{1}{\Gamma(\gamma+1)}\left(\frac{b c \gamma+a c(1-\gamma)}{b c+a d-a c}\right) .
\end{aligned}
$$

Therefore,

$$
\begin{aligned}
\left|{ }^{\mathrm{c}} D_{0^{+}}^{\delta} T x(t)-{ }^{\mathrm{c}} D_{0^{+}}^{\delta} T \bar{x}(t)\right| & \leq \frac{q\|x-\bar{x}\|}{\Gamma(1-\delta)} \int_{0}^{t} \frac{(t-s)^{-\delta}(b c \gamma+a c(1-\gamma))}{\Gamma(\gamma+1)(b c+a d-a c)} d s \\
& \leq \frac{q\|x-\bar{x}\|}{\Gamma(2-\delta) \Gamma(\gamma+1)}\left(\frac{b c \gamma+a c(1-\gamma)}{b c+a d-a c}\right) \\
& \leq q\|x-\bar{x}\| L^{*} .
\end{aligned}
$$

From equations (11) and (12) we obtain that

$$
\|T x-T \bar{x}\| \leq q\|x-\bar{x}\| L+q\|x-\bar{x}\| L^{*} \leq q\|x-\bar{x}\|\left(L+L^{*}\right) .
$$

Also, it is clear that, for each $t \in[-\tau, 0],|T x(t)-T \bar{x}(t)|=0$. Hence, by the Banach contraction principle BVP (1) has a unique solution.

\section{Examples}

In this section, we present some examples to explain the applicability of the main results.

Example 1 Consider the following BVP of FFDE:

$$
D_{0^{+}}^{\gamma} x(t)=\frac{\Gamma(\delta+1)}{64}\left(e^{t}-1\right)+\frac{\Gamma(\delta+1)}{120} e^{-t}\left|x_{t}\right|^{\psi_{1}}+\frac{e^{-t}}{143}\left|D_{0^{+}}^{\delta} x_{t}\right|^{\psi_{2}}, \quad 0<t<1,
$$

with boundary conditions

$$
\begin{array}{ll}
x(t)+2 x^{\prime}(t)=\zeta(t), & -\tau \leq t \leq 0, \\
3 x(t)-\frac{1}{2} x^{\prime}(t)=\eta(t), & 0 \leq t \leq 1,
\end{array}
$$

where $\zeta(0)=\eta(1)=0, D_{0^{+}}^{\gamma}$ and $D_{0^{+}}^{\delta}$ are the Caputo derivative with $1<\gamma<2$ and $0<\delta<1$, $t \in(0,1)$. Take $m(t)=\frac{\Gamma(\delta+1)}{64}\left(e^{t}-1\right), j=\frac{\Gamma(\delta+1)}{120} e^{-t}, k=\frac{e^{-t}}{143}$. So

$$
f\left(t, x_{t}, D_{0^{+}}^{\delta} x_{t}\right):=\frac{\Gamma(\delta+1)}{64}\left(e^{t}-1\right)+\frac{\Gamma(\delta+1)}{120} e^{-t}\left|x_{t}\right|^{\psi_{1}}+\frac{e^{-t}}{143}\left|D_{0^{+}}^{\delta} x_{t}\right|^{\psi_{2}} .
$$

Then, for $t \in(0,1)$, we obtain

$$
\left|f\left(t, x_{t}, D_{0^{+}}^{\delta} x_{t}\right)\right| \leq m(t)+j\left|x_{t}\right|^{\psi_{1}}+k\left|D_{0^{+}}^{\delta} x_{t}\right|^{\psi_{2}}
$$

For $0<\psi_{1}, \psi_{2}<1,(\mathrm{C} 1)$ is satisfied. Similarly, for $\psi_{1}, \psi_{2}>1$, (C2) of Theorem 3.1 is proved. Therefore, BVP (13) has a solution. 
Example 2 Consider BVP for the fractional functional differential equation

$$
D_{0^{+}}^{\frac{3}{2}} x(t)=\frac{\left|x_{t}\right|+\left|D_{0^{+}}^{\frac{1}{2}} x_{t}\right|}{\left(12+15 e^{2 t}\right)\left(1+\left|x_{t}\right|+\mid D_{0^{+}}^{\frac{1}{2}} x_{t}\right) \mid}
$$

with boundary conditions

$$
\begin{aligned}
& x(0)+2 x^{\prime}(0)=0, \\
& 3 x(1)-\frac{1}{2} x^{\prime}(1)=0 .
\end{aligned}
$$

Here $\gamma=\frac{3}{2}, \delta=\frac{1}{2}$, and

$$
f\left(t, x_{t}, D_{0^{+}}^{\delta} x_{t}\right)=\frac{\left|x_{t}\right|+\left|D_{0^{+}}^{\frac{1}{2}} x_{t}\right|}{\left(12+15 e^{2 t}\right)\left(1+\left|x_{t}\right|+\left|D_{0^{+}}^{\frac{1}{2}} x_{t}\right|\right)} .
$$

Take

$$
f\left(t, x_{1}, x_{2}\right)=\frac{\left|x_{1}\right|+\left|x_{2}\right|}{\left(12+15 e^{2 t}\right)\left(1+\left|x_{1}\right|+\left|x_{2}\right|\right)}
$$

and consider $x_{1}, x_{2}, \bar{x}_{1}, \bar{x}_{2} \in C_{\tau}$. Then, for every $t \in[0,1]$,

$$
\begin{aligned}
\left|f\left(t, x_{1}, x_{2}\right)-f\left(t, \bar{x}_{1}, \bar{x}_{2}\right)\right| & =\frac{1}{12+15 e^{2 t}}\left|\frac{\left|x_{1}\right|+\left|x_{2}\right|}{1+\left|x_{1}\right|+\left|x_{2}\right|}-\frac{\left|\bar{x}_{1}\right|+\left|\bar{x}_{2}\right|}{1+\left|\bar{x}_{1}\right|+\left|\bar{x}_{2}\right|}\right| \\
& \leq \frac{1}{12+15 e^{2 t}}\left(\frac{\left|x_{1}-\bar{x}_{1}\right|+\left|x_{2}-\bar{x}_{2}\right|}{\left(1+\left|x_{1}\right|+\left|x_{2}\right|\right)\left(1+\left|\bar{x}_{1}\right|+\left|\bar{x}_{2}\right|\right)}\right) \\
& \leq \frac{1}{12+15 e^{2 t}}\left(\left|x_{1}-\bar{x}_{1}\right|+\left|x_{2}-\bar{x}_{2}\right|\right) \\
& \leq \frac{1}{27}\left(\left|x_{1}-\bar{x}_{1}\right|+\left|x_{2}-\bar{x}_{2}\right|\right) .
\end{aligned}
$$

For every $t \in[-\tau, 0]$, we have

$$
\begin{aligned}
\left|f\left(t, x_{1}, x_{2}\right)-f\left(t, \bar{x}_{1}, \bar{x}_{2}\right)\right| & =\frac{1}{12+15 e^{2 t}}\left|\frac{\left|x_{1}\right|+\left|x_{2}\right|}{1+\left|x_{1}\right|+\left|x_{2}\right|}-\frac{\left|\bar{x}_{1}\right|+\left|\bar{x}_{2}\right|}{1+\left|\bar{x}_{1}\right|+\left|\bar{x}_{2}\right|}\right| \\
& \leq \frac{1}{12+15 e^{2 t}}\left(\frac{\left|x_{1}-\bar{x}_{1}\right|+\left|x_{2}-\bar{x}_{2}\right|}{\left(1+\left|x_{1}\right|+\left|x_{2}\right|\right)\left(1+\left|\bar{x}_{1}\right|+\left|\bar{x}_{2}\right|\right)}\right) \\
& \leq \frac{1}{12}\left(\left|x_{1}-\bar{x}_{1}\right|+\left|x_{2}-\bar{x}_{2}\right|\right) .
\end{aligned}
$$

Furthermore,

$$
\begin{aligned}
L & =\frac{1}{\Gamma(\gamma+1)}\left[\frac{a d(1-\gamma)+\gamma b d}{b c+a d-a c}\right] \\
& =\frac{1}{\Gamma\left(\frac{3}{2}+1\right)}\left[\frac{a d\left(1-\frac{3}{2}\right)+\frac{3}{2} b d}{b c+a d-a c}\right] \\
& \approx 0.268,
\end{aligned}
$$




$$
\begin{aligned}
L^{*} & =\frac{1}{\Gamma(2-\delta) \Gamma(\gamma+1)}\left[\frac{b c \gamma+a c(1-\gamma)}{b c+a d-a c}\right] \\
& =\frac{1}{\Gamma\left(\frac{3}{2}\right) \Gamma\left(\frac{3}{2}+1\right)}\left[\frac{\frac{3}{2} b c+a c\left(1-\frac{3}{2}\right)}{b c+a d-a c}\right] \\
& \approx 1.82 .
\end{aligned}
$$

Also, $\left(L+L^{*}\right)^{-1} \approx 0.478$. This means that $q=0.037<0.083<\left(L+L^{*}\right)^{-1}$. Hence, by Theorem (3.2), BVP (14) has a unique solution.

\section{Competing interests}

The authors declare that they have no competing interests.

\section{Authors' contributions}

All authors read and approved the final manuscript.

\section{Author details}

${ }^{1}$ School of Mathematical Sciences, Anhui University, Hefei, Anhui 230039, China. ${ }^{2}$ Department of Mathematics, School of Natural Sciences (SNS), National University of Sciences and Technology (NUST), H-12 Sector, Islamabad, 44000, Pakistan.

\section{Acknowledgements}

This research had been supported by National Natural Science Foundation of China (Nos. 11371027, 11471015, and 11601003).

Received: 12 November 2016 Accepted: 14 January 2017 Published online: 25 January 2017

\section{References}

1. Kilbas, A, Srivastava, H, Trujillo, J: Theory and Applications of Fractional Differential Equations. Elsevier, Amsterdam (2006)

2. Miller, K, Ross, B: An Introduction to the Fractional Calculus and Fractional Differential Equation. Wiley, New York (1993)

3. Oldham, K, Spanier, J: The Fractional Calculus. Academic Press, New York (1974)

4. Podlubny, I: Fractional Differential Equations. Academic Press, San Diego (1999)

5. Samko, S, Kilbas, A, Marichev, O: Fractional Integral and Derivative: Theory and Applications. Gordon \& Breach, Yverdon (1993)

6. Ahmad, B, Ntouyas, SK: Existence results for a coupled system of Caputo type sequential fractional differential equations with nonlocal integral boundary conditions. Appl. Math. Comput. 266, 615-622 (2015)

7. Eloe, PW, Henderson, J: Uniqueness implies existence and uniqueness conditions for nonlocal boundary value problems for nth order differential equation. J. Math. Anal. Appl. 331, 240-247 (2007)

8. Agarwal, RP, Benchohra, M, Hamani, A: Boundary value problems for fractional differential equations. Georgian Math. J. 16, 401-411 (2009)

9. Diethelm, K: The Analysis of Fractional Differential Equations. Springer, Berlin (2010)

10. Feng, W, Sun, S, Han, Z, Zhao, Y: Existence of solutions for a singular system of nonlinear fractional differential equations. Comput. Math. Appl. 62(3), 1370-1378 (2011)

11. Rehman, $M$, Khan, $R$, Asif, $N$ : Three point boundary value problems for nonlinear fractional differential equations. Acta Math. Sci. Ser. B 31(4), 1337-1346 (2011)

12. Sun, $\mathrm{S}, \mathrm{ZhaO}, \mathrm{Y}, \mathrm{Han}, \mathrm{Z}, \mathrm{Xu}, \mathrm{M}$ : Uniqueness of positive solutions for boundary value problems of singular fractional differential equations. Inverse Probl. Sci. Eng. 20, 299-309 (2012)

13. Sun, S, Zhao, Y, Han, Z, Li, Y: The existence of solutions for boundary value problem of fractional hybrid differential equations. Commun. Nonlinear Sci. Numer. Simul. 17, 4961-4967 (2012)

14. Agarwal, RP, Zhou, Y, He, Y: Existence of fractional neutral functional differential equations. Comput. Math. Appl. 59 , 1095-1100 (2010)

15. Benchohra, M, Henderson, J, Ntouyas, S, Ouahab, A: Existence results for fractional order functional differential equations with infinite delay. J. Math. Anal. Appl. 338, 1340-1350 (2008)

16. Lakshmikantham, V: Theory of fractional functional differential equations. Nonlinear Anal. 69, 3337-3343 (2008)

17. Lakshmikantham, V, Vatsala, A: Basic theory of fractional differential equations. Nonlinear Anal. 69, 2677-2682 (2008)

18. Lakshmikantham, V, Vatsala, A: Theory of fractional differential inequalities and applications. Commun. Appl. Anal. 11 395-402 (2007)

19. Lakshmikantham, V, Devi, J: Theory of fractional differential equations in Banach space. Eur. J. Pure Appl. Math. 1, 38-45 (2008)

20. Guo, D, Lakshmikantham, V: Nonlinear Problems in Abstract Cone. Academic Press, Orlando (1988)

21. Li, Y, Jiang, W: Fractional order nonlinear systems with delay in iterative learning control. Appl. Math. Comput. 257, 546-552 (2015)

22. Li, Y, Sun, S, Yang, D, Han, Z: Three-point boundary value problems of fractional functional differential equations with delay. Bound. Value Probl. 2013, 38 (2013)

23. Li, X, Liu, S, Jiang, W: Positive solutions for boundary value problem of nonlinear fractional functional differential equations. Appl. Math. Comput. 217, 9278-9285 (2011) 
24. Liu, S, Li, X, Zhou, XF, Jiang, W: Synchronization analysis of singular dynamical networks with unbounded time-delays. Adv. Differ. Equ. 2015, 193 (2015)

25. Wen, Y, Zhou, XF, Zhang, Z, Liu, S: Lyapunov method for nonlinear fractional differential systems with delay. Nonlinear Dyn. 82, 1015-1025 (2015)

26. Zhou, XF, Yang, F, Jiang, W: Analytic study on linear neutral fractional differential equations. Appl. Math. Comput. 257, 295-307 (2015)

27. Zhao, K, Wang, K: Existence of solutions for the delayed nonlinear fractional functional differential equations with three-point integral boundary value conditions. Adv. Differ. Equ. 2016, 284 (2016)

28. Xu, S, Radenovic, S: Fixed point theorems of generalized Lipschitz mappings on cone metric spaces over Banach algebras without assumption of normality. Fixed Point Theory Appl. 2014, 102 (2014)

29. Zhang, S: Positive solution for boundary value problem of nonlinear fractional differential equations. Electron. J. Differ. Equ. 2006, 36 (2006)

30. Smart, DR: Fixed Point Theorems. Cambridge University Press, Cambridge (1973)

Submit your manuscript to a SpringerOpen ${ }^{\circ}$ journal and benefit from:

- Convenient online submission

- Rigorous peer review

- Immediate publication on acceptance

- Open access: articles freely available online

- High visibility within the field

- Retaining the copyright to your article 